\title{
Formation of nanoscale polarized clusters as precursors of electronic ferroelectricity probed by conductance noise spectroscopy
}

\author{
Jens Müller, ${ }^{1,2,{ }^{*}}$ Satoshi Iguchi $\odot,{ }^{1}$ Hiromi Taniguchi $\odot,{ }^{3}$ and Takahiko Sasaki ${ }^{1}$ \\ ${ }^{1}$ Institute for Materials Research, Tohoku University, Sendai 980-8577, Japan \\ ${ }^{2}$ Institute of Physics, Goethe University Frankfurt, 60438 Frankfurt (M), Germany \\ ${ }^{3}$ Graduate School of Science and Engineering, Saitama University, Saitama 338-8570, Japan
}

(Received 19 March 2020; accepted 11 September 2020; published 29 September 2020)

\begin{abstract}
We investigate the low-frequency charge-carrier dynamics of a molecular dimer-Mott insulator $\beta^{\prime}$-(BEDT$\mathrm{TTF})_{2} \mathrm{ICl}_{2}$, where the freezing of charge fluctuations on the dimers gives rise to electronic ferroelectricity. We show that conductance fluctuation (noise) spectroscopy allows one to probe changes in the dielectric properties at elevated temperatures, where samples are even still in the conductive regime. Our results explain the formation of electric polarization states leading to glassy and relaxor-type ferroelectric behavior that is frequently observed in these systems. The onset of distinct two-level fluctuations and changes of the underlying $1 / f$-type noise indicate the formation of nanoscale polar regions, the dynamics of which depends on the applied electric fields. Conductance noise spectroscopy therefore is a suitable tool for investigating the onset of electric-polarization dynamics in molecular and other, inorganic charge-driven ferroelectrics.
\end{abstract}

DOI: 10.1103/PhysRevB.102.100103

The notion of a novel type of ferroelectricity, where the polar state is controlled by the electronic degrees of freedom (of charge, spin, and orbital nature) instead of the shifts of atomic positions as in conventional displacive ferroelectrics, recently has become the subject of intensive research efforts including the search for new materials [1-6]. Particularly promising candidates for such electronic ferroelectricity are members of the family of molecular dimerMott insulators (BEDT-TTF) ${ }_{2} X$ [7] based on the organic donor molecule BEDT-TTF (abbreviated as ET) - denoting bis-(ethylenedithio)tetrathiafulvalene, $\mathrm{C}_{6} \mathrm{~S}_{8}\left[\left(\mathrm{CH}_{2}\right)_{2}\right]_{2}$ - and a charge-compensating monovalent anion $X$, where the ferroelectric properties are caused by charge disproportionation in a modulated or bond-alternated dimerized lattice [8-12]. The centrosymmetric dimer-Mott state consists of one $\pi$-hole carrying spin $1 / 2$ whose center of gravity is localized in the center of the molecular dimer unit $(\mathrm{ET})_{2}$ (charge-centered/averaged phase). If the charge, however, becomes shifted towards one of the molecules within the dimer (chargeordered phase), this disproportionation of the charges is the origin of a quantum electric dipole [9,13-16]. The much lighter mass of electrons in charge-driven as opposed to ions in displacive-type ferroelectrics not only may allow considerably faster switching processes, but also causes large temporal and spatial dielectric fluctuations which may play a crucial role in the dielectric and optical properties and the ferroelectric phase transition [17].

In this work, we apply current noise spectroscopy aiming to probe the effects of collective fluctuations also at temperatures far above the Curie-Weiss temperature and the onset of electric polarization, where the samples often are too conductive

*j.mueller@physik.uni-frankfurt.de for performing dielectric spectroscopy (see [18]). We aim to provide evidence for an important consequence of large dielectric fluctuations, namely, the formation of polar nanoregions (PNRs) and electronic (e.g., paraelectric-ferroelectric) phase separation [17], which may help to explain the up-tonow puzzling occurrence of either long-range ferroelectric order or relaxor-type ferroelectricity $[9,10,19]$. In general, albeit a macroscopic probe, noise measurements are suitable to infer microscopic information enabling us to estimate the size of emergent clusters, thus gaining information not only on the ground state, but also on the fluctuations to the excited state, as well as the time and length scales at which the fluctuations occur. This is of fundamental importance for nonuniform electronic or magnetic states in condensed-matter systems.

For the present study, we have chosen $\beta^{\prime}-(\mathrm{ET})_{2} \mathrm{ICl}_{2}$ (in short, $\beta^{\prime}-\mathrm{ICl}_{2}$ ), a strong dimer-Mott insulator (Fig. 1), where electric-field-induced charge disproportionation is demonstrated in charge-sensitive Raman scattering [20]. We consider $\beta^{\prime}-\mathrm{ICl}_{2}$ a model system for studying the underlying charge fluctuations on the dimers in a square lattice arrangement [see Fig. 2(f) and the Supplemental Material [18]], where-in contrast to spin-liquid-like properties in some triangularlattice systems - both the ground state and the excited states are expected to be simpler. We aim to elucidate the emergence of the charge fluctuations and their freezing in a material with metastable dipolar states, where the ground state is a Mott insulator, and address yet unanswered questions of the appearance and stabilization of polar clusters/domains and their response to electric fields. The system shows relaxorlike ferroelectric behavior with a strongly frequency-dependent peak of the dielectric constant developing below $T \sim 125 \mathrm{~K}$ and a Curie-Weiss temperature of $T_{C}=67 \mathrm{~K}$ [21]. Below about $60 \mathrm{~K}$, pyrocurrent measurements reveal the onset of electricfield-induced macroscopic polarization [21]. The $P$ - $E$ curves 


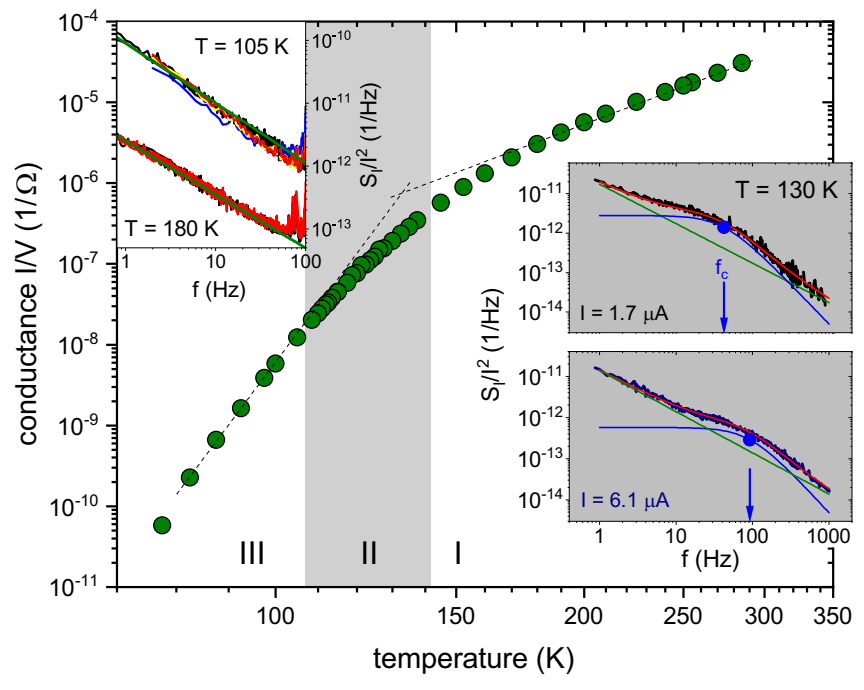

FIG. 1. Conductance of $\beta^{\prime}-\mathrm{ICl}_{2}$ vs temperature. (Dashed lines are guides to the eye indicating a crossover to more insulating behavior at about 130-140 K.) In the areas labeled I and III the normalized current noise PSD is $1 / f$-like and independent of the applied electric field (left inset; green lines are fits to $S_{I} / I^{2} \propto 1 / f^{\alpha}$; for $T=180 \mathrm{~K}$, two sample currents $I=1.68$ and $1.95 \mu \mathrm{A}$ are shown, and for $T=105 \mathrm{~K}$ four currents $I=0.189,0.281,0.326$, and $0.371 \mu \mathrm{A})$. In the gray-shaded temperature regime II $(110 \mathrm{~K} \lesssim T \lesssim 140 \mathrm{~K})$, a single Lorentzian is superimposed on the $1 / f$-type "backgound" noise and $S_{I} / I^{2}(f)$ depends on the external electric field. Right insets show representative spectra for regime II. Lines are fits to Eq. (1), where $\alpha$ has been set to a value of 1 .

showing a small value of only a few percent of the fully polarized moment and relaxation effects in Raman spectroscopy [20] suggest a dipole-glass state at low temperatures. We apply conductance fluctuation (noise) spectroscopy, which has proven a sensitive tool to study glassy structural and chargecarrier dynamics in (ET) $)_{2} X$ systems [22-25], and is capable of detecting fluctuating entities on the nano-/mesoscopic scale [26,27].

We have measured in total four single crystals of $\beta^{\prime}$-(ET) ${ }_{2} \mathrm{ICl}_{2}$ showing qualitatively similar results. The data shown here are obtained on a sample with dimensions $(0.1 \times 0.23 \times 1.92) \mathrm{mm}^{3}$ along the $a, b$, and $c$ directions. Conductance measurements have been performed along the crystallographic $c$ axis.

Figure 1 shows the de conductance $G=V / I$ vs temperature $T$ of $\beta^{\prime}-\mathrm{ICl}_{2}$, where $V$ is the voltage applied across the sample and $I$ the measured electric current flow. [For reference: the conductivity at $T=285 \mathrm{~K}$ amounts to $\sigma=9.4 \times$ $10^{-3}(\Omega \mathrm{cm})^{-1}$.] The $\log G$ vs $\log T$ representation indicates a crossover to more insulating behavior (stronger decrease of the conductance) below about 130-140 K (see dashed lines in Fig. 1). An analysis of $G(T)$ in terms of different transport models is given in the Supplemental Material [18]. At discrete temperatures, the current noise power spectral density (PSD) $S_{I}(f, T)$ [28] has been recorded for various excitation voltages $V$, which allows one to identify three different temperature regimes as follows. The normalized noise spectra are of generic $1 / f^{\alpha}$ type and independent of the applied electric field $\mathcal{E}=V / l$ (with $l$ being the distance between the electric contacts along the sample's $c$ axis), i.e., the expected scaling $S_{I} \propto I^{2}$ is obeyed [29], for temperatures $T \gtrsim 140 \mathrm{~K}$ (regime I) and $T \lesssim 110 \mathrm{~K}$ (regime III). Representative noise spectra in these regimes are shown in the left inset of Fig. 1. In between, for $110 \mathrm{~K} \lesssim T \lesssim 140 \mathrm{~K}$ (regime II), we find a single Lorentzian spectrum - characteristic for a fluctuating two-level system — superimposed on the "background":

$$
\frac{S_{I}(f, T)}{I^{2}}=\frac{a}{f^{\alpha}}+\frac{b}{f^{2}+f_{c}^{2}},
$$

where $a(T)$ and $b(T)$ are the amplitudes of the $1 / f$-type and Lorentzian noise contributions, respectively. $\alpha(T)$ denotes the frequency exponent of the $1 / f$ noise, and $f_{c}(T)$ the corner frequency of the two-level fluctuator. Note that in our frequency window of $100 \mathrm{mHz}-1 \mathrm{kHz}, b(T) \neq 0$ only in regime II. Strikingly, for these temperatures the observed normalized noise spectra depend on $\mathcal{E}$, i.e., the $S_{I} \propto I^{2}$ scaling is not valid in regime II. $f_{c}$ is related to the characteristic energy of the fluctuator and for thermally activated states of a double-well potential one has

$$
f_{c}=f_{0} \exp \left(-\frac{E_{a}}{k_{B} T}\right)
$$

with an activation energy $E_{a}$, an attempt frequency $f_{0}$, and Boltzmann's constant $k_{B}$ [30].

The anomalous current dependence of the noise spectra in regime II is shown exemplarily in the right inset of Fig. 1, where the excellent quality of the fits to Eq. (1) is demonstrated. Clearly, $f_{c}$ and hence the characteristic energy of the fluctuation process, shift with $I$, i.e., depend on the electric field $\mathcal{E}$ : at $T=130 \mathrm{~K}, f_{c}$ increases from $42 \mathrm{~Hz}$ for a sample current of $I=1.7 \mu \mathrm{A}$ to $f_{c}=93 \mathrm{~Hz}$ for $I=6.1 \mu \mathrm{A}$ (blue arrows).

This intriguing and unexpected current/electric field dependence of the noise spectra in the temperature regime II is illustrated in more detail for a representative temperature of $T=116.5 \mathrm{~K}$ in Fig. 2(a). Solid lines are fits to Eq. (1), whereat in the representation $f \times S_{I} / I^{2}$ vs $f$, the $1 / f$ term is a constant (for $\alpha=1$ ) and the Lorentzian term peaks at $f_{c}$ (see the respective dotted and dashed curves representing these contributions for two selected currents). One main result of this study, displayed in Fig. 2(b), is a threshold behavior of the corner frequency $f_{c}$, which stays roughly constant for small values of the electric field $\mathcal{E}$ (orange arrow) until it increases exponentially above a threshold field $\mathcal{E}_{t h}$ (red arrow). Thus, a distinct low- $\mathcal{E}$ and high- $\mathcal{E}$ behavior is observed. Phenomenologically, we describe the characteristic frequency of the two-level fluctuations by Eq. (2) for $\mathcal{E} \leqslant \mathcal{E}_{t h}$ and by an additional term (see also [26]) resulting in

$$
f_{c}=f_{0} \exp \left(\frac{p\left(\mathcal{E}-\mathcal{E}_{t h}\right)-E_{a}}{k_{B} T}\right) \text { for } \mathcal{E} \geqslant \mathcal{E}_{t h} .
$$

We note that for all applied electric fields shown here, the $I-V$ characteristics [Fig. 2(c)] remain linear, which excludes a trivial heating effect to account for the shift of $f_{c}$. Considering first the roughly constant subthreshold value $f_{c}^{0} \equiv f_{c}(\mathcal{E} \rightarrow 0)$, we find an excellent fit to an Arrhenius behavior, Eq. (2), yielding $E_{a} / k_{B}=(2790 \pm 70) \mathrm{K}$ [see Fig. 2(d)]. This energy 

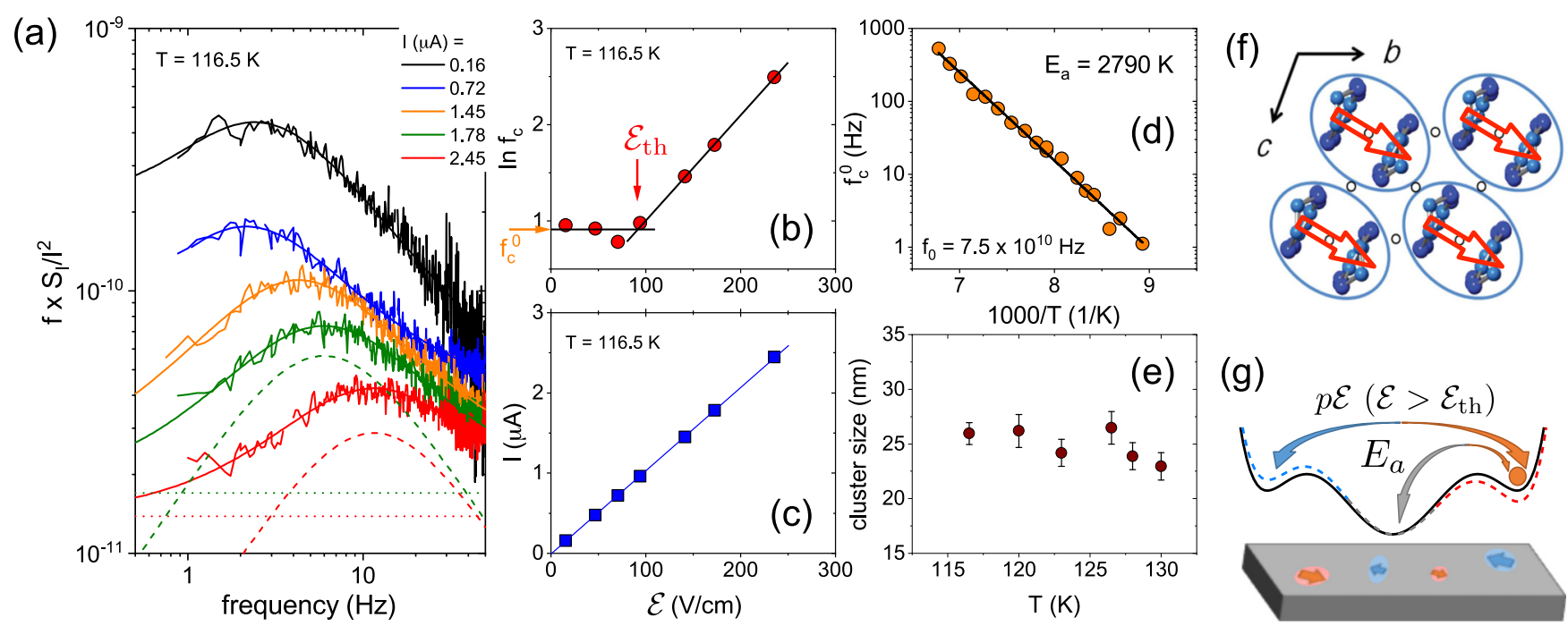

FIG. 2. (a) Representative noise spectra as $f \times S_{I} / I^{2}$ vs $f$ in temperature regime II at fixed $T=116.5 \mathrm{~K}$ and different sample currents as indicated. Smooth solid lines are fits to Eq. (1). For two selected currents, the $1 / f$ and Lorentzian contributions are shown (dotted and dashed lines, respectively). (b) Electric field dependence of the corner frequency $f_{c}$. Lines are guides for the eyes indicating the threshold field $\mathcal{E}_{t h}$ and the zero-field value $f_{c}^{0} \equiv f_{c}\left(\mathcal{E} \rightarrow 0\right.$ ). (c) demonstrates the linear $I-V$ regime. (d) Arrhenius plot of $f_{c}^{0}$ vs $1 / T$ revealing an activation energy $E_{a}=2790 \mathrm{~K}$. (e) Cluster size calculated from $p$ estimated from Eq. (3) (see text). (f) Arrangement of the ET dimers in the conducting planes and orientation of the electric dipoles. (g) Schematics of the suggested zero-field/electric-field-induced dielectric/ferroelectric fluctuations (see text for details).

is very close to the intradimer transfer integral and optical charge gap of $\sim 2900 \mathrm{~K}$ [31-33], which indicates that the observed switching processes in temperature regime II are of electronic origin. We suggest that the competing interand intradimer Coulomb interactions [14,15] cause coherent fluctuations of electrons within a cluster of dimers (i.e., the gravity center of the hole), switching between the unpolarized dimer-Mott state and a charge disproportionated state [see scheme $\left[E_{a}\right]$ in Fig. $\left.2(\mathrm{~g})\right]$. This is in marked contrast to a recent suggestion [34] that the dipolar moments on the dimers are caused by the freezing of the ET molecules' terminal ethylene groups in particular configurations, thereby breaking the local inversion symmetry.

We next analyze the above-threshold behavior following Eq. (3). The constant slope $p$, that can be determined from a linear fit to the data in Fig. 2(b), indicates a discrete value of a fluctuating dipole moment that corresponds to a cluster of a certain size. At $T=116.5 \mathrm{~K}$ we find $p=1.73 \times 10^{-23} \mathrm{C} \mathrm{cm}$ and a threshold field of $\mathcal{E}_{t h} \sim 92 \mathrm{~V} / \mathrm{cm}$. With the dipole moment $p_{d}=0.13 e d$ parallel to the $b$ axis estimated in [21] $[e$ and $d=3.6 \AA$ are the electron charge and distance between the ET molecules in a dimer, respectively; see Fig. 2(f)], we find that the total number $N$ of elementary dipoles within the cluster-taking into account the projection onto the measured $c$ axis-amounts to $N=p / p_{d}^{c \text { axis }} \approx 9 \times 10^{4}$ dimers. Assuming a spherical object, this corresponds to a fluctuating cluster volume with radius $r_{\text {cluster }} \approx 26 \mathrm{~nm}$. Thus, the observed lowfrequency dynamics below $\sim 140 \mathrm{~K}$ is caused by the formation of fluctuating PNRs, which above a threshold field $\mathcal{E}_{t h}$ undergo switching between two states of polarization $+p$ and $-p$ [see scheme $[p \mathcal{E}]$ in Fig. 2(g)]. This dipole-dimer picture has the two polar states $(+p$ and $-p)$ and one nonpolar state $(n)$ whose energies and corresponding lifetimes become modified in a finite electric field $\mathcal{E}>\mathcal{E}_{t h}$. A simple estimate [35] shows that $f_{c}$ does not change due to the lifetime of the nonpolar dimer-Mott state but rather always increases due to a secondorder effect related to the lifetimes $\tau_{+p}$ and $\tau_{-p}$. This can be viewed as a transition between the $+p$ and $-p$ states and determines the origin of the threshold field $\mathcal{E}_{t h}$. Figure 2(e) shows the estimates of the cluster size for other temperatures revealing a roughly constant or slightly increasing size of the PNR with decreasing temperature.

To complete our picture of the charge-carrier dynamics coupled to the dielectric behavior in $\beta^{\prime}-\mathrm{ICl}_{2}$ probed by current noise, we next discuss the $1 / f^{\alpha}$ "background" fluctuations. Figure 3 shows the normalized noise magnitude $S_{I} / I^{2}(f=$ $1 \mathrm{~Hz}, T)=a(T) / f^{\alpha} \equiv a(T)$ (main panel) and the frequency exponent $\alpha(T)$ (inset) resulting from fits of the spectra at different temperatures to Eq. (1). Note that also for regime II, where Lorentzian spectra are superimposed on the $1 / f$-type fluctuations, only the latter are shown here. However, at these temperatures, also the $1 / f$ noise exhibits a distinct asymptotic low- $\mathcal{E}$ and high- $\mathcal{E}$ behavior, shown in Fig. 3 as blue and red symbols, respectively.

At high temperatures (regime I) the $1 / f$-noise magnitude in the Mott insulating state, where the charge is averaged over the dimer, smoothly increases and the charge transport may be understood in terms of variable range hopping [18]. The frequency exponent $\alpha(T \gtrsim 140 \mathrm{~K}) \approx 0.8$, i.e., there is considerably more spectral weight at higher than at lower frequencies. As discussed above, at about $140 \mathrm{~K}$, upon entering regime II, fluctuating PNRs are formed with one such fluctuator being enhanced in our "noise window," i.e., it strongly couples to the conductance fluctuations, which become a sensitive probe to changes in the dielectric properties. The fluctuating electrical polarization potentials of the PNRs 


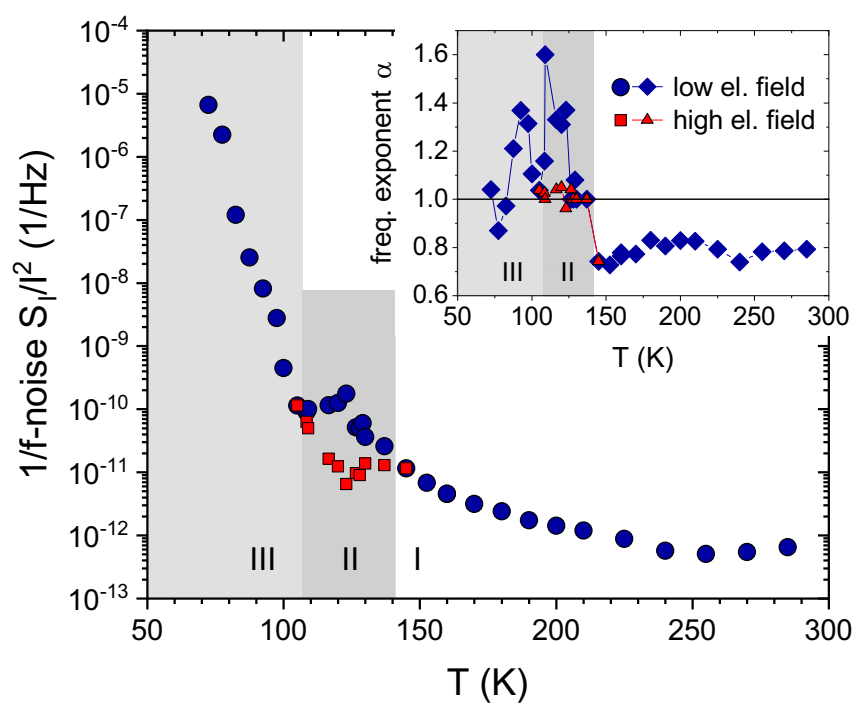

FIG. 3. Normalized current noise PSD $S_{I} / I^{2}$ of $\beta^{\prime}-\mathrm{ICl}_{2}$ taken at $1 \mathrm{~Hz}$ vs $T$. Shown is only the term $a(T)$ in Eq. (1). The inset shows the frequency exponent $\alpha(T)$. Blue and red symbols denote the asymptotic low- and high-electric-field behavior, respectively, in regime II.

act on the transport channel as small capacitor gates that locally modulate the conductance of the underlying resistor network [36,37]. The superposition of many fluctuating PNRs then constitutes part of the $1 / f$-type background. We now first discuss the behavior at low electric fields (blue symbols): the local maximum of the $1 / f^{\alpha}$-noise magnitude $a(T)$ at $T \approx 125 \mathrm{~K}$ reveals the "resonance" of the independently fluctuating PNR (at $f=1 \mathrm{~Hz}$ ). This picture of an enhanced localization of charge carriers and increasingly slow dynamics due to the formation of dimer units that fluctuate coherently as extended clusters is corroborated by the rather abrupt increase of the frequency exponent $\alpha(T)$ from almost constant values of $\alpha(T) \approx 0.8$ in temperature regime I to values of $\alpha \gtrsim 1$ below about $140 \mathrm{~K}$ (see inset of Fig. 3). This behavior implies a substantial shift of the current fluctuations' spectral weight to low frequencies below this temperature, which may indicate some clusters merging and possibly switching cooperatively.

The red data points in Fig. 3 represent the high- $\mathcal{E}$ values. As shown in Fig. 2(a), the $1 / f$-noise magnitude decreases with increasing electric field, with a tendency for saturation at $\mathcal{E} \sim 300 \mathrm{~V} / \mathrm{cm}$ (not shown), with $\alpha$ approaching values of 1. This can be understood by the stabilization of an increasing number and/or size of PNRs that increases the total volume of slow fluctuators, thereby reducing the $1 / f$-type noise in regime II which may be viewed as the superposition of many independently fluctuating clusters with a broad energy distribution [30]. This behavior goes along with a suppression, broadening, and shift of the local maximum of the $1 / f$ noise to higher temperatures corresponding to a positive shift of the characteristic "resonance" frequency (energy) in agreement with the observations for a single fluctuator discussed above. Upon further decreasing the temperature, in regime III the slow dynamics of the now stabilized PNR undergoing ferroelectric fluctuations dominates the $1 / f$ noise, which below about $110 \mathrm{~K}$ shows a drastic increase. This corresponds to a change of the electronic transport mechanism, which can be inferred from the scaling behavior $S_{I} / I^{2}$ vs $G$ (see [18]).

In our picture regime II $(110 \mathrm{~K} \lesssim T \lesssim 140 \mathrm{~K})$ marks the transformation to the ergodic relaxor state, in which polar regions on nanometer scale with randomly distributed directions of dipole moments, the PNR, appear. (In relaxor ferroelectrics, this temperature can be twice as high as their freezing temperature giving rise to the peak in permittivity [38].) This corresponds to the transformation from conductive to dielectric behavior, which is in agreement with the experimental observation that the dielectric response begins to appear in regime II [21], being accompanied by a slowing down of the charge fluctuations seen in the conductance noise. In $\beta^{\prime}$-(ET) $)_{2} \mathrm{ICl}_{2}$, the (low-frequency) noise due to switching of PNRs (in regime II) and the low-frequency dispersion of the dielectric response (in regime I) may have the same origin.

We note that our observation is in accordance with recent studies of Raman scattering [20], where at temperatures of $15 \mathrm{~K}$ and below, i.e., far below the ferroelectric CurieWeiss temperature, additional Raman peaks are induced by an electric field $\mathcal{E}=5 \mathrm{kV} / \mathrm{cm}$ demonstrating intradimer charge disproportionation and indicating a "discrete" polarization of macroscopic domains, where the total $\mathcal{E}$-induced polarization observed at low temperatures amounts to only a few percent of the total sample volume [39], suggesting a glassy polarization [20,21].

In conclusion, our findings demonstrate that conductance noise spectroscopy provides a sensitive tool for probing the formation of PNRs or fluctuating mesoscopic domains and their coupling to electric fields for studying the physics of materials giving rise to electronic ferroelectricity. We suggest to perform similar studies for other relaxor- or long-range-type ferroelectrics at temperatures above the application regime of dielectric spectroscopy. Indeed, first results on the multiferroic system $\kappa-(\mathrm{ET})_{2} \mathrm{Cu}\left[\mathrm{N}(\mathrm{CN})_{2}\right] \mathrm{Cl}[10,19]$ of a sample exhibiting relaxor ferroelectricity [40] show a very similar behavior [18], suggesting that electric-field-induced stabilization of fluctuating PNRs may be a common feature of electronic ferroelectricity in molecular and possibly also inorganic materials.

J.M. acknowledges financial support during his stay at IMR, Tohoku University, Sendai within the ICC-IMR program, and support by the Deutsche Forschungsgemeinschaft (DFG, German Research Foundation) through TRR 288 - 422213477 (project B02). Work at IMR was funded within MEXT/JSPS KAKENHI Grants No. JP18H04298, No. JP16K05430, and No. JP18K18725. Work at IMR was funded within KAKENHI Grants No. 18H04298, No. 16K05430, and No. 18K18725. We thank M. Kurosu and B. Hartmann for help with the noise measurements and are grateful to $\mathrm{K}$ Hashimoto and M. Naka for stimulating discussions. We also thank S. M. Winter for support with quantum chemical calculations of the ethylene endgroup conformations' energies. 
[1] J. van den Brink and D. I. Khomskii, J. Phys.: Condens. Matter 20, 434217 (2008).

[2] S. Horiuchi and Y. Tokura, Nat. Mater. 7, 357 (2008).

[3] S. Ishihara, J. Phys. Soc. Jpn. 79, 011010 (2010)

[4] D. S. Chow, F. Zamborszky, B. Alavi, D. J. Tantillo, A. Baur, C. A. Merlic, and S. E. Brown, Phys. Rev. Lett. 85, 1698 (2000).

[5] T. Kimura, T. Goto, H. Shintani, K. Ishizaka, T. H. Arima, and Y. Tokura, Nature (London) 426, 55 (2003).

[6] N. Ikeda, H. Ohsumi, K. Ohwada, K. Ishii, T. Inami, K. Kakurai, Y. Murakami, K. Yoshii, S. Mori, and Y. Horibe, Nature (London) 436, 1136 (2005).

[7] P. Lunkenheimer and A. Loidl, J. Phys.: Condens. Matter 27, 373001 (2015).

[8] K. Yamamoto, S. Iwai, S. Boyko, A. Kashiwazaki, F. Hiramatsu, C. Okabe, N. Nishi, and K. Yakushi, J. Phys. Soc. Jpn. 77, 074709 (2008).

[9] M. Abdel-Jawad, I. Terasaki, T. Sasaki, N. Yoneyama, N. Kobayashi, Y. Uesu, and C. Hotta, Phys. Rev. B 82, 125119 (2010).

[10] P. Lunkenheimer, J. Müller, S. Krohns, F. Schrettle, A. Loidl, B. Hartmann, R. Rommel, M. de Souza, C. Hotta, J. A. Schlueter, and M. Lang, Nat. Mater. 11, 755 (2012).

[11] K. Kobayashi, S. Horiuchi, R. Kumai, F. Kagawa, Y. Murakami, and Y. Tokura, Phys. Rev. Lett. 108, 237601 (2012).

[12] E. Gati, J. K. H. Fischer, P. Lunkenheimer, D. Zielke, S. Köhler, F. Kolb, H.-A. K. von Nidda, S. M. Winter, H. Schubert, J. A. Schlueter, H. O. Jeschke, R. Valentí, and M. Lang, Phys. Rev. Lett. 120, 247601 (2018).

[13] R. T. Clay, H. Li, and S. Mazumdar, Physica B 405, S253 (2010); H. Li, R. T. Clay, and S. Mazumdar, J. Phys.: Condens. Matter 22, 272201 (2010).

[14] M. Naka and S. Ishihara, J. Phys. Soc. Jpn. 79, 063707 (2010).

[15] C. Hotta, Crystals 2, 1155 (2012).

[16] N. Hassan, S. Cunningham, M. Mourigal, E. I. Zhilyaeva, S. A. Torunova, R. N. Lyubovskaya, J. A. Schlueter, and N. Drichko, Science 360, 1101 (2018).

[17] S. Ishihara, J. Phys.: Condens. Matter 26, 493201 (2014).

[18] See Supplemental Material at http://link.aps.org/supplemental/ 10.1103/PhysRevB.102.100103 for the details of the crystal structure, the experimental setup, the comparison of conductance and impedance spectroscopy measurements, the transport mechanism as deduced from the conductance and noise measurements, and a comparison to the triangular lattice dimer-Mott insulator $\kappa-(\mathrm{ET})_{2} \mathrm{Cu}\left[\mathrm{N}(\mathrm{CN})_{2}\right] \mathrm{Cl}$. The Supplemental Material includes Refs. [41-47].

[19] M. Lang, P. Lunkenheimer, J. Müller, A. Loidl, B. Hartmann, N. H. Hoang, E. Gati, H. Schubert, and J. A. Schlueter, IEEE Trans. Magn. 50, 2700107/1 (2014).

[20] Y. Hattori, S. Iguchi, T. Sasaki, S. Iwai, H. Taniguchi, and H. Kishida, Phys. Rev. B 95, 085149 (2017).

[21] S. Iguchi, S. Sasaki, N. Yoneyama, H. Taniguchi, T. Nishizaki, and T. Sasaki, Phys. Rev. B 87, 075107 (2013).

[22] J. Müller, ChemPhysChem 12, 1222 (2011).

[23] F. Kagawa, T. Sato, K. Miyagawa, K. Kanoda, Y. Tokura, K. Kobayashi, R. Kumai, and Y. Murakami, Nat. Phys. 9, 419 (2013)

[24] J. Müller, B. Hartmann, R. Rommel, J. Brandenburg, S. M. Winter, and J. A. Schlueter, New J. Phys. 17, 083057 (2015).

[25] S. Sasaki, K. Hashimoto, R. Kobayashi, K. Itoh, S. Iguchi, Y. Nishio, Y. Ikemoto, T. Moriwaki, N. Yoneyama, M. Watanabe,
A. Ueda, H. Mori, K. Kobayashi, R. Kumai, Y. Murakami, J. Müller, and T. Sasaki, Science 357, 1381 (2017).

[26] B. Raquet, A. Anane, S. Wirth, P. Xiong, and S. von Molnár, Phys. Rev. Lett. 84, 4485 (2000).

[27] J. Müller, J. Brandenburg, and J. A. Schlueter, Phys. Rev. Lett. 102, 047004 (2009).

[28] If the fluctuating part of the signal is $\delta I(t)=I(t)-\langle I(t)\rangle$, where $\langle I(t)\rangle$ is the time-averaged mean value that may be considered equal to zero, the noise PSD is calculated by $S_{I}(f)=$ $2 \lim _{T \rightarrow \infty}\left|\int_{-T / 2}^{T / 2} \delta I(t) e^{-2 \pi f t} d t\right|^{2}[29]$.

[29] J. Müller and T. Thomas, Crystals 8, 166 (2018).

[30] B. Raquet, Electronic Noise in Magnetic Materials and Devices, Spin Electronics, edited byM. Ziese and M. J. Thornton (Springer, Heidelberg, 2001).

[31] T. Koretsune and C. Hotta, Phys. Rev. B 89, 045102 (2014).

[32] K. Hashimoto, R. Kobayashi, H. Okamura, H. Taniguchi, Y. Ikemoto, T. Moriwaki, S. Iguchi, M. Naka, S. Ishihara, and T. Sasaki, Phys. Rev. B 92, 085149 (2015).

[33] N. Tajima, R. Kato, and H. Taniguchi, Europhys. Lett. 83, 27008 (2008).

[34] M. Sawada, S. Fukuoka, and A. Kawamoto, Phys. Rev. B 97, 045136 (2018).

[35] In regime II, the dipoles are not stable without electric field and the dipole-dimer state $(p)$ has a higher energy than the nonpolar dimer-Mott state $(n)$. For $\mathcal{E} \rightarrow 0$ the switching frequency $2 \pi f_{c}=1 / \tau_{\text {eff }}=1 / \tau_{p}+1 / \tau_{n}$ is mostly determined by the shorter lifetime (higher energy) state $p$ rather than the more stable (longer lifetime) state $n$. In a finite electric field $\mathcal{E}>\mathcal{E}_{\text {th }}$, the energies of these states and their corresponding lifetimes $\left(\tau_{+p}, \tau_{-p}\right.$, and $\left.\tau_{n}\right)$ become modified such that $\tau_{+p} \rightarrow\left(\tau_{+p}+\delta t\right)$ and $\tau_{-p} \rightarrow\left(\tau_{-p}-\delta t\right)$ with $\delta t>0$. The change in the characteristic frequency $f_{c}$ of the fluctuating unit then can be evaluated to $2 \pi f_{c}=\frac{2}{\tau_{n}}+\frac{1}{\left(\tau_{+p}+\delta t\right)}+\frac{1}{\left(\tau_{-p}-\delta t\right)} \approx \frac{2}{\tau_{n}}+\frac{2}{\tau}+\frac{2(\delta t)^{2}}{\tau^{3}}$, where we assume $\tau \equiv \tau_{+p} \approx \tau_{-p} \ll \tau_{n}$ and $\delta t \ll \tau$. Thus, the linear term in $\delta t$ cancels out and $f_{c}$ does not change due to the lifetime of the nonpolar dimer-Mott state but rather always increases due to the second-order term $(\delta t)^{2}$ related to the lifetimes $\tau_{+p}$ and $\tau_{-p}$.

[36] B. I. Shklovskii, Phys. Rev. B 67, 045201 (2003).

[37] A. L. Burin, B. I. Shklovskii, V. I. Kozub, Y. M. Galperin, and V. Vinokur, Phys. Rev. B 74, 075205 (2006).

[38] A. A. Bokov and Z.-G. Ye, J. Mater. Sci. 41, 31 (2006); J. Adv. Dielectrics 2, 1241010 (2012).

[39] From $P-E$ curves at $T=10 \mathrm{~K}, 2 \%$ polarization of the total volume was estimated, and about $0.5 \%$ polarization was estimated from pyrocurrent measurements at $2 \mathrm{kV} / \mathrm{cm}$ [21].

[40] T. Thomas, B. Hartmann, P. Lunkenheimer, H. Schubert, J. A. Schlueter, and J. Müller, Phys. Status Solidi B 256, 1800746 (2019).

[41] K. Sano, T. Sasaki, N. Yoneyama, and N. Kobayashi, Phys. Rev. Lett. 104, 217003 (2010).

[42] D. Stauffer and A. Aharony, Introduction to Percolation Theory (Taylor \& Francis, London, 1992).

[43] Sh. Kogan, Electronic Noise and Fluctuations in Solids (Cambridge University Press, Cambridge, UK, 1996).

[44] A.-M. S. Tremblay, S. Feng, and P. Breton, Phys. Rev. B 33, 2077 (1986).

[45] C. C. Chen and Y. C. Chou, Phys. Rev. Lett. 54, 2529 (1985).

[46] U. N. Nandi, C. D. Mukherjee, and K. K. Bardhan, Phys. Rev. B 54, 12903 (1996).

[47] J. Planès and A. François, Phys. Rev. B 70, 184203 (2004). 